\title{
Surgical treatment of pyoderma gangrenosum following deep inferior epigastric perforator flap breast reconstruction
}

\author{
Funda Tamer ${ }^{1 凶}$, Esra Adışen², Serhan Tuncer ${ }^{3}$, Mehmet A. Gurer ${ }^{2}$
}

\begin{abstract}
Pyoderma gangrenosum is a chronic inflammatory disease characterized by painful cutaneous ulcers. The etiology remains unknown; however, pyoderma gangrenousm can be triggered by surgery. Here we report the case of a 34-year-old Caucasian female that developed pyoderma gangrenosum following deep inferior epigastric perforator flap breast reconstruction. The patient was successfully treated with systemic immunosuppressive therapy and primary closure.
\end{abstract}

Keywords: Pyoderma gangrenosum, surgery

Received: 14 June 2016 | Returned for modification: 13 July 2016 | Accepted: 19 July 2016

\section{Introduction}

Pyoderma gangrenosum (PG) is a rare noninfectious neutrophilic dermatosis characterized by recurrent cutaneous ulcerations (1). The disease often appears on the pretibial area. However, it can affect the head and neck region, trunk, breast, hand, and peristomal skin (2). The diagnosis of PG is usually made by excluding other causes of cutaneous ulcers (3). Pyoderma gangrenosum may present with some systemic diseases including ulcerative colitis, Crohn's disease, hepatitis $\mathrm{C}$, seronegative rheumatoid arthritis, spondylitis, monoclonal gammopathies, leukemia, lymphoma, and myelodysplastic syndrome. Therefore, patients should be evaluated for any underlying disease (4). Pyoderma gangrenosum may occur after trauma or injury to the skin. This condition is called pathergy. Pathergy phenomenon is positive in $30 \%$ of patients with PG. Therefore, PG can be triggered by many surgical procedures (5).

\section{Case report}

A 34-year-old Caucasian female complaining of cutaneous ulcers was admitted to our dermatology department. She had a past medical history of breast cancer treated with total mastectomy of the right breast. Therefore she underwent deep inferior epigastric perforator flap breast reconstruction at the department of plastic and reconstructive surgery. One week after surgery, she had evident erythema, seropurulent drainage, and pain in the area around the incision for the reconstructed breast and the abdomen. Wound and blood culture were both negative. On dermatological examination, there was erythema on the right breast surrounding an open wound with fibrinous debris and limited granulation tissue. We also examined ulceration with mucopurulent and hemorrhagic exudate at the abdominal incision site. The ulcer borders were undermined, bluish, and surrounded by erythema (Figs. $1 \mathrm{a}-\mathrm{b})$. Histological examination of the lesions on the patient's right breast and abdomen revealed a dermal-epidermal neutrophilic infiltrate with abscess, ulcer formation, and superficial vessels with fibrin deposition (Fig. 1c). The diagnosis of PG was made based on the appearance of skin lesions, negative wound culture, and histopathology report. The patient was started on a course of high-dose methylprednisolone (64 mg/day p.o.). After 8 weeks of steroid therapy, the treatment was still unsatisfactory. Therefore, cyclosporine $(5 \mathrm{mg} / \mathrm{kg} /$ day p.o.) was added to the treatment regimen and the steroid dose was gradually reduced. The patient achieved a partial response despite receiving cyclosporine for 4 months. Thus she underwent primary repair at the plastic surgery department (Figs. 2a-b).

Her postoperative period was uneventful. The ulcerative lesions almost completely healed through scar formation over a period of 8 months. Therefore the corticosteroid and cyclosporine therapy was stopped gradually over the next 7 months. (Fig. 2c).

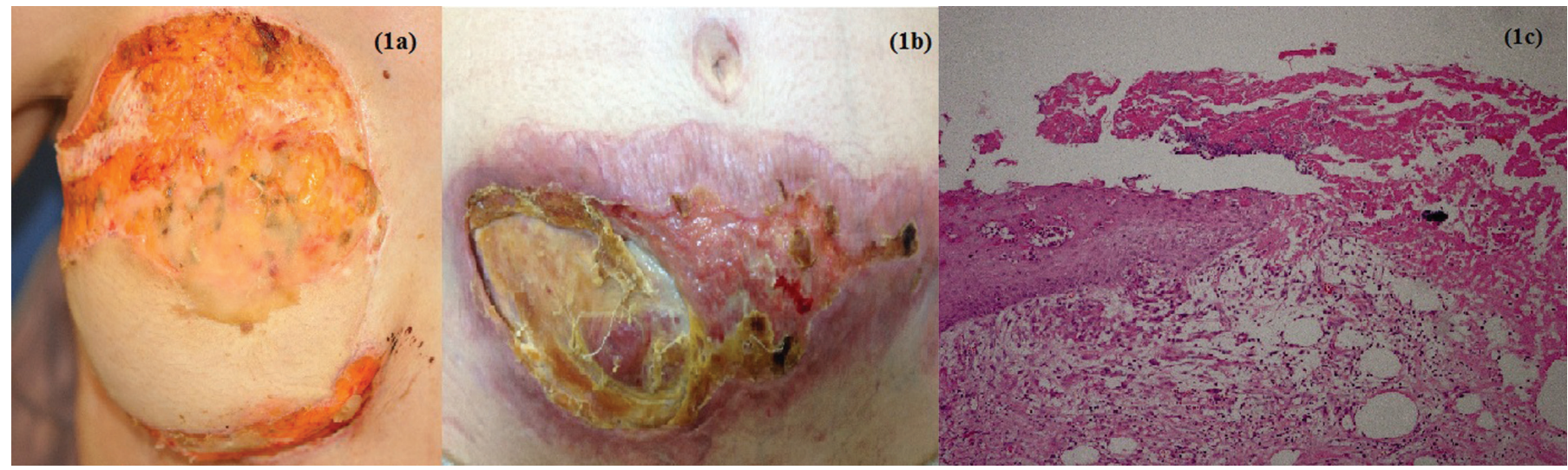

Figure $1 \mid a-b)$ Ulcers on the patient’s right breast and infraumbilical region, $c$ ) Ulceration with granulation tissue (hematoxylin and eosin stain, $\times 100$ )

${ }^{1}$ Medical Park Hospital, Department of Dermatology, Ankara, Turkey. ${ }^{2}$ Gazi University Faculty of Medicine, Department of Dermatology, Ankara, Turkey. ${ }^{3}$ Gazi University Faculty of Medicine, Department of Plastic, Reconstructive and Aesthetic Surgery, Ankara, Turkey. $\bowtie$ Corresponding author: fundatmr@ yahoo.com 


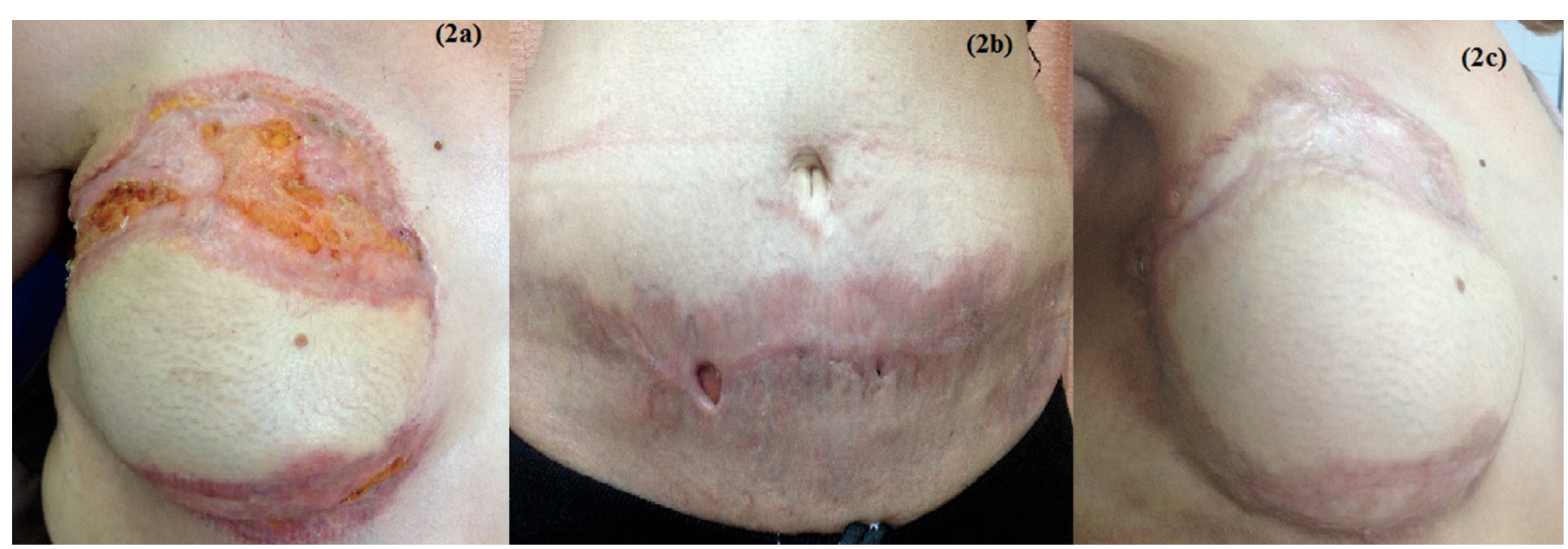

Figure 2 (a-b) Appearance of the lesions after primary closure, c) Scar tissue left by the ulcers.

\section{Discussion}

Pyoderma gangrenosum typically presents with sterile pustules that turn into painful ulcers. The ulcers usually have irregular, undermined, and violaceous colored borders that extend rapidly (2). It has been suggested that PG can be triggered by many surgical procedures as the result of tissue damage. Pyoderma gangrenosum lesions may also mimic surgical wound infections. Misdiagnosis as wound infection can expose patients to unnecessary antibiotic therapies that are not appropriate for initial management (5).

The aim of therapy is to modulate the immune system and reduce the inflammatory process. Systemic treatments include corticosteroids, cyclosporine, azathioprine, intravenous immunoglobulin, infliximab, and etanercept (6). Surgery should be avoided if possible. The pathergy phenomenon that may occur with surgical procedures can result in wound enlargement. Most reported cases of PG treated with surgical procedures have had poor patient outcomes. Nonetheless, there have been reports of patients treated successfully with gentle sharp debridement, skin grafting, and reconstruction. Chen et al. reported a patient with vulvar PG that was successfully managed through surgical debridement and primary closure without flap reconstruction (7).

Our case presented above details the successful treatment course of a patient that developed extensive PG after breast reconstruction. In our case, surgery accelerated the wound healing course and reduced potential complications of immunosuppressive therapy. We suggest that primary closure may be performed for patients with PG without recurrence in combination with immunosuppressant medication such as systemic corticosteroids and cyclosporine.

\section{References}

1. Spear M. Pyoderma gangrenosum: an overview. Plast Surg Nurs. 2008;28:154-7.

2. Wollina U. Pyoderma gangrenosum-a review. Orphanet J Rare Dis. 2007;2:19.

3. Ruocco E, Sangiuliano S, Gravina AG, Miranda A, Nicoletti G. Pyoderma gangrenosum: an updated review. J Eur Acad Dermatol Venereol. 2009;23:1008-17.

4. Cunha VS, Mango V, Wendt LRG, Furian TQ, Passos EP. Pyoderma gangrenosum postlaparoscopy: a rare complication. Surg Laparosc Endosc Percutan Tech. 2012;22:45-7.

5. Caterson SA, Nyame T, Phung T, Lee B, Tobias AM. Pyoderma gangrenosum following bilateral deep inferior epigastric perforator flap breast reconstruction. J Reconstr Microsurg. 2010;26:475-9.

6. Miller J, Yentzer BA, Clark A, Jorizzo JL, Feldman SR. Pyoderma gangrenosum: a review and update on new therapies. J Am Acad Dermatol. 2010;62:646-54.

7. Chen JR, Chen SS, Chan YJ. Rapid recovery of vulvar pyoderma gangrenosum in response to aggressive surgery and steroid treatment. Taiwan J Obstet Gynecol. 2014:53:97-100. 